\title{
Damping of oscillations by ion-neutral collisions in a prominence plasma
}

\author{
P. Forteza ${ }^{1}$, R. Oliver ${ }^{1}$, J. L. Ballester ${ }^{1}$, and M. L. Khodachenko ${ }^{2}$ \\ 1 Departament de Física, Universitat de les Illes Balears, 07122 Palma de Mallorca, Spain \\ e-mail: [pep. forteza; ramon.oliver; dfsjlbQ]@uib.es \\ 2 Space Research Institute, Austrian Academy of Sciences, 8042 Graz, Austria \\ e-mail: maxim. khodachenko@oeaw.ac.at
}

Received 26 June 2006 / Accepted 29 September 2006

ABSTRACT

\begin{abstract}
Aims. The role of collisions between ions, electrons and neutrals in a partially ionised plasma is assessed as a possible wave damping mechanism. The relevance of this mechanism in the damping of small amplitude prominence oscillations is evaluated.

Methods. A one-fluid MHD set of equations taking into account various effects in a partially ionised solar plasma (collisions between different species and Joule dissipation) is derived. Assuming small perturbations, these equations are next linearised about a uniform equilibrium configuration and the dispersion relation of magnetoacoustic waves in an unbounded medium is obtained.

Results. The presence of neutrals in the plasma only affects the fast wave in a relevant way. An approximate expression for the damping rate is obtained which shows that the strongest damping takes place in a medium with strong magnetic field, low density and low ionisation fraction. Wave attenuation arises mostly from collisions between ions and neutrals.

Conclusions. Given the poor knowledge about the values of the density and ionisation fraction in prominences, it is hard to judge the importance of the physics of partial ionisation in the damping of fast waves in solar prominences. Nevertheless, note that a very idealised case, with no stratification and no equilibrium currents, is considered here, so the addition of these features to the model may change the results of this work.
\end{abstract}

Key words. magnetohydrodynamics (MHD) - Sun: prominences - Sun: oscillations

\section{Introduction}

Prominences are relatively cool objects embedded in the hotter corona, with temperature one hundred times smaller and density one hundred times larger than that of the coronal medium. These objects are observed in $\mathrm{H} \alpha$ as bright features above the solar limb and as thin, dark ribbons, called filaments, on the disc. They often appear as a sheet of plasma with a long horizontal dimension (length $\sim 10^{5} \mathrm{~km}$, width $\sim 10^{4} \mathrm{~km}$ ). The composition of the prominences is not well known, but it seems that the abundance of helium is smaller than $10 \%$ and the abundance of hydrogen is higher than $90 \%$. Other elements are present in the prominence plasma, although in very small quantities. In addition to this, the prominence material seems to be partially ionised, but the ionisation degree of prominences varies over a wide range (with the ratio of electron to neutral hydrogen density roughly between 0.1 and 10, see Patsourakos \& Vial 2002). In connection with this issue, it is worth mentioning the recent advances in the non-LTE modelling of solar prominences (e.g. Labrosse \& Gouttebroze 2004; Anzer \& Heinzel 2005; Heinzel et al. 2005; Chiuderi Drago 2005), which can shed some light on their intimate structure.

One of the important goals of the study of prominences is to develop models that explain how such cool, dense clouds can exist in the surrounding hot, tenuous coronal plasma. These models assume that prominences are supported against gravity by the coronal magnetic field. In general, models of prominences invoke a support mechanism for the prominence material that involves an upward magnetic force, which balances the downward gravitational force. However, if the prominence plasma is only partially ionised one may wonder how the neutral component of the plasma is supported. For a plausible solution to this problem see Gilbert et al. (2002).

A possible path for a deeper understanding of prominences is to exploit their oscillatory nature. From the observational point of view, prominence oscillations can be grossly classified, according to the amplitude of periodic variations, in large- and small-amplitude oscillations. The analysis of data provided by time series of several indicators (line width, line intensity and Doppler velocity) has permitted to establish that small-amplitude prominence oscillations are of local nature and to classify them in long-period oscillations ( $T>40 \mathrm{~min}$ ), intermediate-period (10 $\mathrm{min}<T<40 \mathrm{~min}$ ) and short-period $(T<10 \mathrm{~min})$, although this classification does not reflect the origin or nature of the perturbations causing the different periods. On the other hand, so far there is little information about the wavelength, phase speed and lifetime of oscillations, which are of remarkable interest for a detailed comparison with theoretical models of prominence vibrations. Recent reviews of the topic can be found in Oliver (1999), Engvold (2001) and Oliver \& Ballester (2002).

The existence of various dissipative mechanisms (e.g. viscosity, thermal and electric conductivity, radiative cooling, etc.) can result in the dissipation of the wave energy, which is consequently absorbed by the medium and hence the wave amplitude is progressively attenuated. The damping mechanisms considered in Ballai (2003), for spatial damping, and Terradas et al. (2001) and Carbonell et al. (2004), for temporal damping, only 
allow to explain the damping of slow waves, so it is necessary to take into account other damping mechanisms in order to explain the attenuation of fast waves. The frictional damping of magnetoacoustic waves in a partially ionised plasma is much stronger than in a fully ionised plasma because the presence of neutral atoms causes the Joule dissipation to increase as a result of collisions of electrons with neutrals and ions and, what is more important, of collisions of ions with neutrals (Khodachenko et al. 2004). A comparative study of the role of ion-neutral damping of MHD waves and their damping due to viscosity and thermal conductivity has made by Khodachenko et al. (2004) and Khodachenko et al. (2006) and it was found that collisional damping is dominant. It is well known that the role of neutrals can be very relevant in a cold $(T \sim 10000 \mathrm{~K})$ plasma, such as has been recently found by Leake \& Arber (2006) in the context of the magnetic flux emergence from the solar interior into the corona. These authors find that the chromospheric partial ionisation leads to an increased rate of flux emergence and, more importantly, that the magnetic field becomes force-free after crossing the chromosphere.

The aim of the present work is to study the effect of collisions between ions, electrons and neutrals in the damping of the oscillations of a partially ionised prominence. In Sect. 2 we introduce the basic set of equations for a partially ionised plasma; in Sect. 3 we obtain the dispersion relations for a unbounded, uniform medium in the linear regime; in Sect. 4 the equations derived in Sect. 3 are applied to a prominence plasma and the results are presented; Sect. 5 presents a comparison with the work of Braginskii (1965); finally, in Sect. 6 our conclusions are drawn.

\section{Partially ionised plasma: one-fluid MHD equations}

Let us consider a partially ionised hydrogen plasma with $T_{\alpha}, \rho_{\alpha}$, $n_{\alpha}, p_{\alpha}$ and $\boldsymbol{V}_{\alpha}$ respectively representing the temperature, density, number density, pressure and velocity of the different plasma species: ions $(\alpha=\mathrm{i})$, neutrals $(\alpha=\mathrm{n})$ and electrons $(\alpha=\mathrm{e})$. Obviously, in a hydrogen plasma the number density of ions and electrons is equal, i.e. $n_{\mathrm{e}}=n_{\mathrm{i}}$. Moreover, $\rho_{\alpha}=m_{\alpha} n_{\alpha}$, with $m_{\alpha}$ the particle mass.

The total density, total pressure and centre of mass velocity are $\rho=\rho_{\mathrm{e}}+\rho_{\mathrm{i}}+\rho_{\mathrm{n}} \approx \rho_{\mathrm{n}}+\rho_{\mathrm{i}}, p=p_{\mathrm{e}}+p_{\mathrm{i}}+p_{\mathrm{n}}=2 p_{\mathrm{i}}+p_{\mathrm{n}}$ and

$\boldsymbol{V}=\frac{\Sigma_{\alpha=\mathrm{e}, \mathrm{i}, \mathrm{n}} \rho_{\alpha} \boldsymbol{V}_{\alpha}}{\rho} \approx \xi_{\mathrm{i}} \boldsymbol{V}_{\mathrm{i}}+\xi_{\mathrm{n}} \boldsymbol{V}_{\mathrm{n}}$,

where the assumptions $\rho_{\mathrm{e}} V_{\mathrm{e}} \ll \rho_{\mathrm{i}} V_{\mathrm{i}}$ and $\rho_{\mathrm{e}} V_{\mathrm{e}} \ll \rho_{\mathrm{n}} V_{\mathrm{n}}$ have been made. Here the relative densities of neutrals and ions are defined as

$\xi_{\mathrm{i}}=\frac{\rho_{\mathrm{i}}}{\rho} \approx \frac{n_{\mathrm{i}}}{n_{\mathrm{i}}+n_{\mathrm{n}}}, \quad \xi_{\mathrm{n}}=\frac{\rho_{\mathrm{n}}}{\rho} \approx \frac{n_{\mathrm{n}}}{n_{\mathrm{i}}+n_{\mathrm{n}}}$.

The degree of ionisation of the plasma is characterized by the ionisation fraction, $\tilde{\mu}$, defined as the mean atomic weight (the average mass per particle in units of $m_{\mathrm{p}}$ ). Then,

$\tilde{\mu}=\frac{1}{1+\xi_{\mathrm{i}}}$,

which implies that $\tilde{\mu}=0.5$ for a fully ionised plasma and $\tilde{\mu}=1$ for a neutral gas. Although we retain the relative densities of ions and neutrals in the following, they can be expressed in terms of $\tilde{\mu}$ as $\xi_{\mathrm{i}}=1 / \tilde{\mu}-1$ and $\xi_{\mathrm{n}}=2-1 / \tilde{\mu}$.
In this work we do not include the effects of particle ionisation and recombination, and also assume a strong thermal coupling between the species, which leads electrons, ions and neutrals to have the same temperature $\left(T_{\mathrm{e}}=T_{\mathrm{i}}=T_{\mathrm{n}}=T\right)$. Then, it makes no sense to consider separate continuity, momentum and energy equations for the three components. The separate governing equations for the three species can be easily substituted by a set of one-fluid equations for the whole partially ionised plasma. We now outline the derivation of these expressions, where isotropic pressure has been assumed and gravity, viscosity, heat conduction and non-adiabatic effects have been neglected; CGS units are used throughout.

1. The continuity equation can be obtained by adding the individual continuity equations for $\rho_{\alpha}$, which yields

$$
\frac{\partial \rho}{\partial t}+\nabla \cdot(\rho \boldsymbol{V})=0
$$

2. To derive the momentum equation we start from the respective equations for each species (Eqs. (2.2e) and (2.2i) in Braginskii (1965) for electrons and ions, and a similar one for neutrals). These formulae contain a term that accounts for the transfer of momentum between species; in this work this effect arises from particle collisions and can be treated as in p. 277 of Braginskii (1965). Now the three momentum equations are added and the electron inertia term is neglected in comparison with that of ions and neutrals. Thus, we obtain

$\rho \frac{\mathrm{d} \boldsymbol{V}}{\mathrm{d} t}=-\nabla p+\frac{1}{c} \boldsymbol{j} \times \boldsymbol{B}-\nabla \cdot\left(\xi_{\mathrm{n}} \xi_{\mathrm{i}} \rho \boldsymbol{w} \boldsymbol{w}\right)$,

where $\mathrm{d} / \mathrm{d} t=\partial / \partial t+\boldsymbol{V} \cdot \nabla, \boldsymbol{j}=c /(4 \pi) \nabla \times \boldsymbol{B}$ and $\boldsymbol{w}=\boldsymbol{V}_{\mathrm{i}}-$ $\boldsymbol{V}_{\mathrm{n}}$. The term containing $\boldsymbol{w}$ in Eq. (5), which is caused by the species inertia, is usually neglected in solar applications and also vanishes in the linear regime. An expression for $\boldsymbol{w}$ is given below.

3. To derive the energy equation the starting point are the individual energy equations as given by Braginskii (1965) or in the more convenient form of Eq. (2.63) of Goedbloed \& Poedts (2004). These equations are added and, after some simplifications, we get

$$
\frac{\mathrm{d} p}{\mathrm{~d} t}+\gamma p \nabla \cdot \boldsymbol{V}=(\gamma-1) q_{\text {Joule }}
$$

where the Joule heating term, $q_{\text {Joule }}$, is discussed below. Only Joule dissipation is considered in our treatment, although other mechanisms can be included in this formula.

4. The equation of state is only needed to determine the temperature from $n$ and $p$. It is given by the sum of the equations of state of electrons, ions and neutrals,

$p=n k_{\mathrm{B}} T=\left(2 n_{\mathrm{i}}+n_{\mathrm{n}}\right) k_{\mathrm{B}} T$.

5. The generalised Ohm's law plays a crucial role in the derivation of the induction equation and $q_{\text {Joule }}$ of a partially ionised plasma. Our first step is to calculate an expression for $\boldsymbol{w}$ by adding the momentum equations of electrons and ions multiplied by $\xi_{\mathrm{n}}$ and the momentum equation of neutrals multiplied by $-\xi_{i}$. The result is

$\boldsymbol{w}=-\frac{\boldsymbol{G}}{\alpha_{\mathrm{n}}}+\frac{\xi_{\mathrm{n}}}{c \alpha_{\mathrm{n}}} \boldsymbol{j} \times \boldsymbol{B}+\frac{\alpha_{\mathrm{en}}}{\alpha_{\mathrm{n}}} \frac{\boldsymbol{j}}{e n_{\mathrm{i}}}-\frac{\xi_{\mathrm{n}} \xi_{\mathrm{i}}}{\alpha_{\mathrm{n}}} \rho\left(\frac{d_{\mathrm{i}} \boldsymbol{V}_{\mathrm{i}}}{d t}-\frac{d_{\mathrm{n}} \boldsymbol{V}_{\mathrm{n}}}{d t}\right)$,

with $\mathrm{d}_{\alpha} / \mathrm{d} t=\partial / \partial t+\boldsymbol{V}_{\alpha} \cdot \nabla$. Moreover, $\alpha_{\mathrm{n}}$ and $\alpha_{\mathrm{en}}$ are friction coefficients (whose expressions can be found in the literature, e.g. 
Braginskii 1965; Khodachenko et al. 2004; Leake et al. 2005) and $\boldsymbol{G}$ is the pressure function (Braginskii 1965),

$\boldsymbol{G}=\xi_{\mathrm{n}} \nabla\left(p_{\mathrm{e}}+p_{\mathrm{i}}\right)-\xi_{\mathrm{i}} \nabla p_{\mathrm{n}}$.

Taking into account that the partial pressures of the different species are

$p_{\mathrm{e}}=p_{\mathrm{i}}=\frac{\xi_{\mathrm{i}}}{1+\xi_{\mathrm{i}}} p, \quad p_{\mathrm{n}}=\frac{\xi_{\mathrm{n}}}{1+\xi_{\mathrm{i}}} p$,

the pressure function can be cast as

$\boldsymbol{G}=2 \xi_{\mathrm{n}} \nabla\left(\frac{\xi_{\mathrm{i}}}{1+\xi_{\mathrm{i}}} p\right)-\xi_{\mathrm{i}} \nabla\left(\frac{\xi_{\mathrm{n}}}{1+\xi_{\mathrm{i}}} p\right)$

The next step in the derivation of Ohm's law is to use $\boldsymbol{j}=$ $-e n_{\mathrm{i}}\left(\boldsymbol{V}_{\mathrm{e}}-\boldsymbol{V}_{\mathrm{i}}\right)$ to write $\boldsymbol{V}_{\mathrm{e}}=\boldsymbol{V}+\boldsymbol{\xi}_{\mathrm{n}} \boldsymbol{w}-\boldsymbol{j} /\left(e n_{\mathrm{i}}\right)$. Now the term $\boldsymbol{E}+\boldsymbol{V}_{\mathrm{e}} \times \boldsymbol{B} / c$ is isolated from the momentum equation for the electrons and $\boldsymbol{V}_{\mathrm{e}}$ is eliminated using the previous expression with $w$ given by Eq. (8), where the term in this formula containing derivatives of $\boldsymbol{V}_{\mathrm{i}}$ and $\boldsymbol{V}_{\mathrm{n}}$ can be neglected. This results in

$$
\begin{aligned}
\boldsymbol{E}^{*} \equiv \boldsymbol{E}+\frac{\boldsymbol{V} \times \boldsymbol{B}}{c}= & \frac{\varepsilon \boldsymbol{G}-\nabla p_{\mathrm{e}}}{e n_{\mathrm{i}}}+\frac{\boldsymbol{j}}{\sigma}+\frac{1-2 \varepsilon \xi_{\mathrm{n}}}{e n_{\mathrm{i}} c} \boldsymbol{j} \times \boldsymbol{B} \\
& +\frac{\xi_{\mathrm{n}}}{c \alpha_{\mathrm{n}}}\left[\boldsymbol{G} \times \boldsymbol{B}-\frac{\xi_{\mathrm{n}}}{c}(\boldsymbol{j} \times \boldsymbol{B}) \times \boldsymbol{B}\right],
\end{aligned}
$$

where $\sigma$ is the conductivity and $\varepsilon=\alpha_{\mathrm{en}} / \alpha_{\mathrm{n}}$.

6. To write the induction equation we take the curl of Eq. (12) and insert it into Maxwell's equation

$\nabla \times \boldsymbol{E}=-\frac{1}{c} \frac{\partial \boldsymbol{B}}{\partial t}$

so one can obtain the general form of the induction equation,

$$
\begin{aligned}
\frac{\partial \boldsymbol{B}}{\partial t}= & \nabla \times(\boldsymbol{V} \times \boldsymbol{B})-\frac{c}{e} \nabla \times\left(\frac{\varepsilon \boldsymbol{G}-\nabla p_{\mathrm{e}}}{n_{\mathrm{i}}}\right)-\nabla \times(\eta \nabla \times \boldsymbol{B}) \\
& -\frac{c}{4 \pi e} \nabla \times\left[\frac{1-2 \varepsilon \xi_{\mathrm{n}}}{n_{\mathrm{i}}}(\nabla \times \boldsymbol{B}) \times \boldsymbol{B}\right]-\nabla \times\left(\frac{\xi_{\mathrm{n}}}{\alpha_{\mathrm{n}}} \boldsymbol{G} \times \boldsymbol{B}\right) \\
& +\nabla \times\left\{\frac{\xi_{\mathrm{n}}^{2}}{4 \pi \alpha_{\mathrm{n}}}[(\nabla \times \boldsymbol{B}) \times \boldsymbol{B}] \times \boldsymbol{B}\right\} .
\end{aligned}
$$

Here $\eta=c^{2} /(4 \pi \sigma)$ is the coefficient of magnetic diffusion. Moreover, the quantity $\xi_{\mathrm{n}}^{2} /\left(4 \pi \alpha_{\mathrm{n}}\right)$ is sometimes written as (Khodachenko et al. 2004; Leake et al. 2005; Khodachenko et al. 2006)

$\frac{\xi_{\mathrm{n}}^{2}}{4 \pi \alpha_{\mathrm{n}}}=\frac{\eta_{\mathrm{C}}-\eta}{|\boldsymbol{B}|^{2}}$,

where $\eta_{\mathrm{C}}$ is Cowling's coefficient of magnetic diffusion defined in a similar way as $\eta$, but with the Cowling electroconductivity $\sigma_{\mathrm{C}}=\sigma /\left(1+\xi_{\mathrm{n}}^{2} B_{0}^{2} \sigma / \alpha_{\mathrm{n}} c^{2}\right)$ instead of $\sigma$.

7. Using Eq. (12), the Joule heating term in Eq. (6) can be cast as follows

$$
\begin{aligned}
q_{\text {Joule }} \equiv \boldsymbol{E}^{*} \cdot \boldsymbol{j}= & \frac{\varepsilon \boldsymbol{G}-\nabla p_{\mathrm{e}}}{e n_{\mathrm{i}}} \cdot \boldsymbol{j}+\frac{j^{2}}{\sigma}-\frac{\xi_{\mathrm{n}}}{c \alpha_{\mathrm{n}}}(\boldsymbol{j} \times \boldsymbol{B}) \cdot \boldsymbol{G} \\
& +\frac{\xi_{\mathrm{n}}^{2}}{c^{2} \alpha_{\mathrm{n}}}(\boldsymbol{j} \times \boldsymbol{B})^{2} .
\end{aligned}
$$

Thus, to describe the behaviour of a partially ionised plasma one must consider the set of one-fluid Eqs. (4)-(6) and (14), where the last term in Eq. (5) can be neglected and $q_{\text {Joule }}$ is given by Eq. (16). Where necessary, $p_{\mathrm{e}}$ must be eliminated in favour of $p$ by means of Eq. (10) and $n_{\mathrm{i}}$ in favour of $n$ with the help of $n_{\mathrm{i}}=$ $\xi_{\mathrm{i}} /\left(1+\xi_{\mathrm{i}}\right) n$. Therefore, the plasma variables in these formulae are $\rho, p, \boldsymbol{V}$ and $\boldsymbol{B}$ and so the set of eight scalar equations contains eight scalar dependent variables. The one-fluid MHD equations reduce to their fully ionised, non-ideal counterparts by taking $\xi_{\mathrm{n}}=0, \xi_{\mathrm{i}}=1$. The ideal MHD expressions for an ideal plasma can then be recovered by taking $\eta=0$.

Before proceeding further a careful check of the relative role of all terms in Eq. (14) can be performed. In principle one can neglect the Hall term in the generalised Ohm's law, so the corresponding term (fourth one on the right-hand side of Eq. (14)) vanishes (for details see Leake et al. 2005). In addition, if the temperature and density are relatively smooth spatial functions, so that $\varepsilon, \xi_{\mathrm{i}}$ and $\xi_{\mathrm{n}}$ are constants, the second term on the righthand side of Eq. (14) also disappears.

\section{Linear waves in an unbounded medium}

We consider a uniform plasma with density $\rho_{0}$ and pressure $p_{0}$ permeated by a magnetic field $\boldsymbol{B}_{0}=B_{0} \hat{x}=$ const. Next, small perturbations about this equilibrium are considered (this is justified by our interest in small-amplitude oscillations),

$\boldsymbol{B}=\boldsymbol{B}_{0}+\tilde{\boldsymbol{B}}, \quad p=p_{0}+\tilde{p}, \quad \rho=\rho_{0}+\tilde{\rho}, \quad \boldsymbol{V}=\tilde{\boldsymbol{V}}$.

Now, Eqs. (4)-(6) and (14) are linearised by neglecting the products of perturbed quantities. The Joule heating term does not give any contribution because the electric current in the equilibrium is zero $\left(\boldsymbol{j}_{0}=\mathbf{0}\right)$ since the magnetic field is uniform. Therefore, the resulting changes of state are adiabatic. The set of linear equations obtained is

$\frac{\partial \tilde{\rho}}{\partial t}+\rho_{0} \nabla \cdot \tilde{\boldsymbol{V}}=0$

$\rho_{0} \frac{\partial \tilde{\boldsymbol{V}}}{\partial t}=-\nabla \tilde{p}+\frac{1}{4 \pi}\left[(\nabla \times \tilde{\boldsymbol{B}}) \times \boldsymbol{B}_{0}\right]$,

$\frac{\partial \tilde{p}}{\partial t}+\gamma p_{0} \nabla \cdot \tilde{\boldsymbol{V}}=0$

and

$$
\begin{aligned}
\frac{\partial \tilde{\boldsymbol{B}}}{\partial t}= & \nabla \times\left(\tilde{\boldsymbol{V}} \times \boldsymbol{B}_{0}\right)+\eta \nabla^{2} \tilde{\boldsymbol{B}}-\Xi \nabla \times\left(\nabla \tilde{p} \times \boldsymbol{B}_{0}\right) \\
& +\frac{\eta_{\mathrm{C}}-\eta}{\left|\boldsymbol{B}_{0}\right|^{2}} \nabla \times\left\{\left[(\nabla \times \tilde{\boldsymbol{B}}) \times \boldsymbol{B}_{0}\right] \times \boldsymbol{B}_{0}\right\},
\end{aligned}
$$

with

$\Xi=\frac{\xi_{\mathrm{n}}^{2} \xi_{\mathrm{i}}}{\left(1+\xi_{\mathrm{i}}\right) \alpha_{\mathrm{n}}}$.

We now derive the equations governing the propagation of linear waves in an unbounded medium.

\subsection{Dispersion relation and analytical limit}

We Fourier-analyse the variables as follows,

$\tilde{f}(x, y, z, t)=f \mathrm{e}^{\mathrm{i}\left(\omega t+k_{x} x+k_{z} z\right)}$,

and take motions and propagation in the $x z$-plane (i.e., $V_{y}=0$ and $k_{y}=0$ ). In this way we remove Alfvén waves and study the temporal damping of fast and slow magnetoacoustic waves. 
Upon inserting Eq. (23) into Eqs. (18)-(21) and performing the usual algebraic operations we get the dispersion relation for fast and slow waves,

$k^{2} v_{\mathrm{a}}^{2}\left[c_{\mathrm{s}}^{2}\left(k_{x}^{2}-\mathrm{i} k_{z}^{2} \Xi \rho_{0} \omega\right)-\omega^{2}\right]$

$+\mathrm{i} \omega\left(k^{2} \eta_{\mathrm{C}}+i \omega\right)\left(k^{2} c_{\mathrm{s}}^{2}-\omega^{2}\right)=0$,

where $k^{2}=k_{x}^{2}+k_{z}^{2}$ and the sound and Alfvén speed squared are defined as

$c_{\mathrm{s}}^{2}=\frac{\gamma p_{0}}{\rho_{0}}=\frac{\gamma R T_{0}}{\tilde{\mu}}, \quad v_{\mathrm{a}}^{2}=\frac{B_{0}^{2}}{4 \pi \rho_{0}}$,

where $R$ is the gas constant.

In a fully ionised plasma $\xi_{\mathrm{n}}=0$ and $\eta_{\mathrm{C}}=\eta$, so that the terms introduced by the ion-neutral collision mechanism disappear. Furthermore, if the plasma is ideal $(\sigma \rightarrow \infty)$ then we obtain the dispersion relation for magnetoacoustic waves

$\omega^{4}-k^{2}\left(c_{\mathrm{s}}^{2}+v_{\mathrm{a}}^{2}\right) \omega^{2}+k^{2} k_{x}^{2} c_{\mathrm{s}}^{2} v_{\mathrm{a}}^{2}=0$,

with solutions given by the usual expressions (in the limit $c_{\mathrm{S}} \ll$ $\left.v_{\mathrm{a}}\right)$,

$\omega \approx k v_{\mathrm{a}}$,

$\omega \approx k_{x} c_{\mathrm{s}}$,

for the fast and slow wave, respectively.

We next write $\omega=\omega_{\mathrm{R}}+\mathrm{i} \omega_{\mathrm{I}}$ and calculate approximate expressions for the real and imaginary parts of the frequency. Equation (24) is split into its real and imaginary parts and, considering the case of weak damping $\left(\omega_{\mathrm{I}} \ll \omega_{\mathrm{R}}\right)$, its real part leads to the dispersion relation for magnetoacoustic waves in an ideal, fully ionised plasma (Eq. (26)). This means that the real part of the frequency can be calculated from Eqs. (27). From the imaginary part of the dispersion relation one can obtain an expression for the imaginary part of the frequency, namely

$\omega_{\mathrm{I}} \approx \frac{k^{2} k_{z}^{2} c_{\mathrm{s}}^{2} v_{\mathrm{a}}^{2} \Xi \rho_{0}+\eta_{\mathrm{C}} k^{2}\left(\omega_{\mathrm{R}}^{2}-k^{2} c_{\mathrm{s}}^{2}\right)}{4 \omega_{\mathrm{R}}^{2}-2 k^{2}\left(c_{\mathrm{s}}^{2}+v_{\mathrm{a}}^{2}\right)}$.

Substituting $\omega_{\mathrm{R}}$ from the ideal case into this formula yields

$$
\begin{aligned}
2 \omega_{\mathrm{I}}^{\mathrm{fast}} & =\frac{c^{2} k^{2}}{4 \pi \sigma}+\frac{\xi_{\mathrm{n}}^{2} B_{0}^{2}}{4 \pi \alpha_{\mathrm{n}}}\left(k^{2}+\frac{c_{\mathrm{s}}^{2}}{v_{\mathrm{a}}^{2}} k_{z}^{2} \frac{\xi_{\mathrm{i}}}{1+\xi_{\mathrm{i}}}\right) \\
& \approx \frac{c^{2} k^{2}}{4 \pi \sigma}+\frac{\xi_{\mathrm{n}}^{2} B_{0}^{2}}{4 \pi \alpha_{\mathrm{n}}} k^{2},
\end{aligned}
$$

for fast waves (where the last approximation is valid in the limit $\left.c_{\mathrm{s}} \ll v_{\mathrm{a}}\right)$, and

$2 \omega_{\mathrm{I}}^{\text {slow }}=\frac{c^{2}}{4 \pi \sigma} \frac{c_{\mathrm{s}}^{2}}{v_{\mathrm{a}}^{2}} k_{z}^{2}+\frac{\rho_{0} c_{\mathrm{s}}^{2}}{\alpha_{\mathrm{n}}} \frac{\xi_{\mathrm{n}}^{2}}{1+\xi_{\mathrm{i}}} k_{z}^{2}$,

for slow waves. The factor 2 on the left-hand side of these expressions is included for consistency with Eqs. (8.38), (8.41), (8.44), (8.47b) and (8.49) of Braginskii (1965).

\subsection{Perturbations}

Since we are in the linear regime, to determine the perturbed variables once the frequency has been computed one can impose an arbitrary value to one of them $\left(V_{z}\right.$, say). Then, all other perturbations can be computed from

$V_{x}=\frac{c_{\mathrm{s}}^{2} k_{x} k_{z}}{\omega^{2}-c_{\mathrm{s}}^{2} k_{x}^{2}} V_{z}$
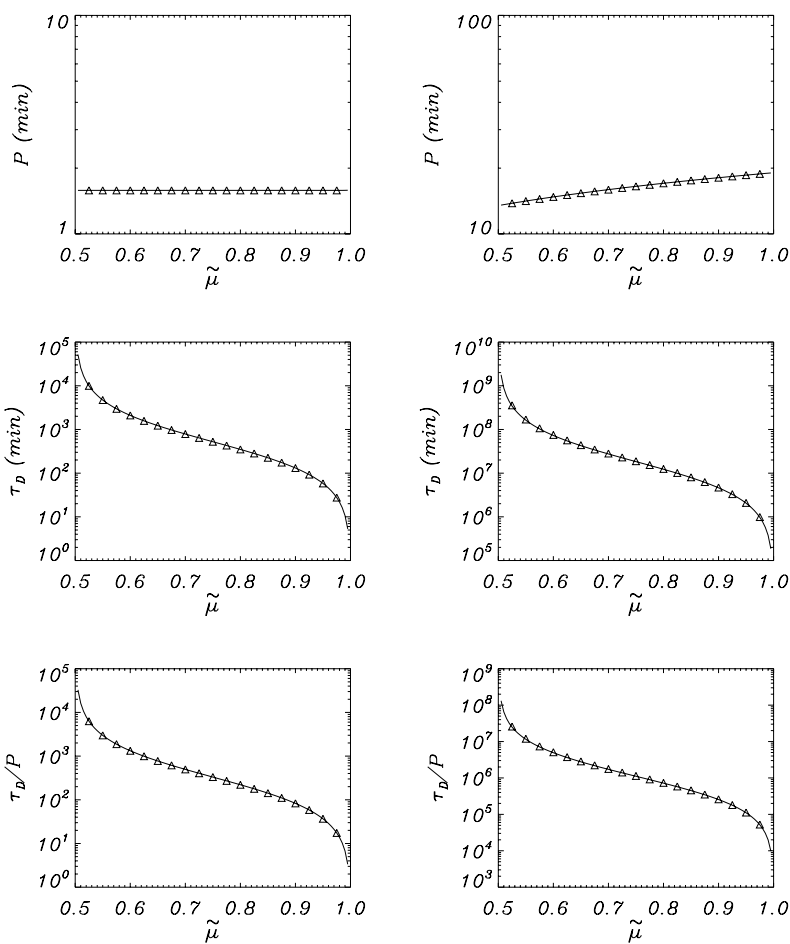

Fig. 1. Period, damping time and $\tau_{\mathrm{D}} / P$ versus the ionisation fraction for the fast wave (left) and the slow wave (right). The parameter values used are $T_{0}=8000 \mathrm{~K}, \rho_{0}=5 \times 10^{-14} \mathrm{~g} / \mathrm{cm}^{3}$ and $B_{0}=10 \mathrm{G}$, for which the Alfvén speed is $126 \mathrm{~km} \mathrm{~s}^{-1}$, the sound speed ranges from 10.5 to $14.9 \mathrm{~km} \mathrm{~s}^{-1}$ and the plasma $\beta$ varies between 0.008 and 0.017 . In addition, $k_{x} x_{0}=\pi / 2$ and $k_{z} x_{0}=0.1$. The solid lines represent the numerical solutions of Eq. (24) while the triangles represent the results obtained with the aproximate Eqs. (27), (29) and (30).

$p=-\frac{\rho_{0} \omega}{k_{x}} V_{x}$,

$B_{z}=\frac{k_{x}\left[B_{0}^{2}\left(\mathrm{i} V_{z}+k_{z} \Xi p\right)+4 \pi\left(\eta-\eta_{\mathrm{C}}\right)\left(k_{z} p+\rho_{0} \omega V_{z}\right)\right]}{B_{0}\left(k^{2} \eta+i \omega\right)}$,

$B_{x}=\frac{B_{0} k_{x} B_{z}-4 \pi\left(k_{z} p+\rho_{0} \omega V_{z}\right)}{B_{0} k_{z}}$.

\section{Results for a prominence plasma}

In this work we concentrate on the features of magnetoacoustic waves in a partially ionised prominence plasma. Physical quantities with their units are used, except for the components of the wavenumber, which are given as $k_{x} x_{0}$ and $k_{z} x_{0}$ (with $x_{0}$ a typical prominence scale-length, for example the prominence width; here $x_{0}=3000 \mathrm{~km}$ ). We first solve Eq. (24) and represent the results in Fig. 1, where the values of $T_{0}, \rho_{0}, B_{0}, k_{x}$ and $k_{z}$ have been fixed in order to study the variation of the pe$\operatorname{riod}\left(P=2 \pi / \omega_{\mathrm{R}}\right)$, the damping time $\left(\tau_{\mathrm{D}}=1 / \omega_{\mathrm{I}}\right)$ and the ratio of the damping time to the period $\left(\tau_{\mathrm{D}} / P\right)$ of magnetoacoustic waves with the ionisation fraction. The values of the damping time reveal that ion-neutral collisions, which are the main cause for the damping of magnetoacoustic waves in the present scenario, are more important for fast waves (for which $\tau_{\mathrm{D}} / P$ is between 1 and $10^{5}$ ) than for slow waves (for which $\tau_{\mathrm{D}} / P$ varies between $10^{4}$ and $10^{8}$ ). Therefore, one can conclude that the effects arising from the partial ionisation of the plasma are irrelevant regarding the slow wave and that fast waves can be damped efficiently for moderate values of the ionisation fraction (i.e. $\tilde{\mu}$ close 

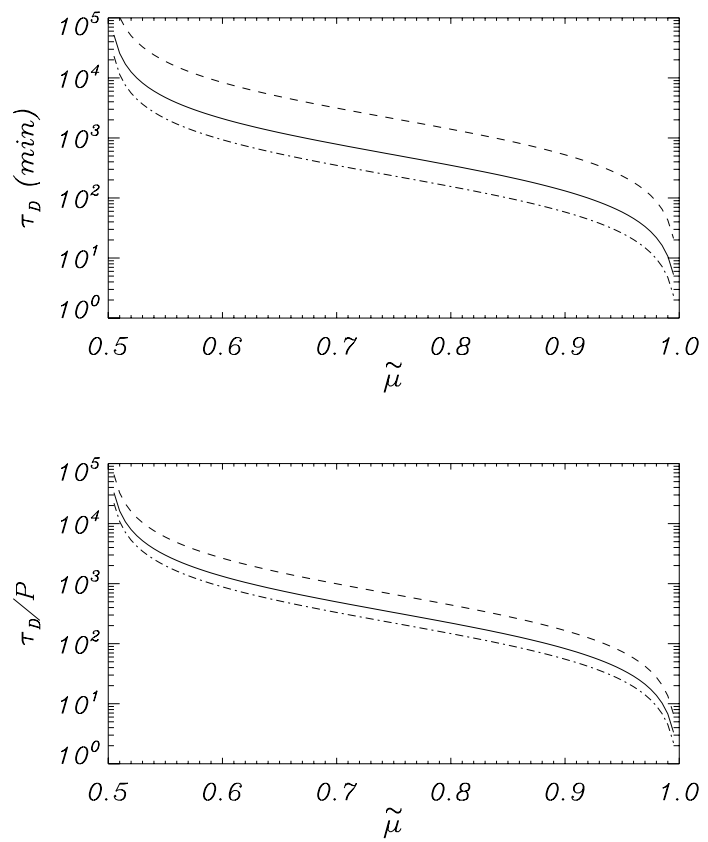

Fig. 2. Fast wave damping time and $\tau_{\mathrm{D}} / P$ versus the ionisation fraction for different values of the magnetic field: $B_{0}=5 \mathrm{G}$ (dashed), $B_{0}=10 \mathrm{G}$ (solid) and $B_{0}=15 \mathrm{G}$ (dash-dotted). The other parameters are $T_{0}=$ $8000 \mathrm{~K}$ and $\rho_{0}=5 \times 10^{-14} \mathrm{~g} / \mathrm{cm}^{3}$, so that the Alfvén and sound speed vary in the ranges $63-189 \mathrm{~km} \mathrm{~s}^{-1}$ and $10.5-14.9 \mathrm{~km} \mathrm{~s}^{-1}$, respectively. Moreover, $k_{x} x_{0}=\pi / 2$ and $k_{z} x_{0}=0.1$.

to 1). The tiny damping rate found when the plasma is nearly fully ionised $(\tilde{\mu} \sim 0.5)$ is a consequence of the small amount of neutrals present in the plasma, which are insufficient to damp the perturbations.

The triangles in Fig. 1 correspond to the solutions obtained using the approximate expresions (27), (29) and (30), which have been derived in the low $\beta$ limit. The perfect agreement between the two results implies that the assumptions made to obtain these approximations are valid and that Eqs. (29) and (30) can be used to obtain some insight into the magnetoacoustic wave features. For example, the fast wave decrement $\omega_{\text {I }}$ can be well approximated by Eq. (29), in which the second term on the right-hand side is dominant so that

$\omega_{\mathrm{I}} \propto \frac{\xi_{\mathrm{n}}^{2}}{1-\xi_{\mathrm{n}}} \rho_{0}^{-1} B_{0}^{2} k^{2}$,

where the factor $\left[\left(1-\xi_{\mathrm{n}}\right) \rho_{0}\right]^{-1}$ comes from $\alpha_{\mathrm{n}}$. Combining this formula with Eq. (27) results in

$\frac{\tau_{\mathrm{D}}}{P} \propto \frac{1-\xi_{\mathrm{n}}}{\xi_{\mathrm{n}}^{2}} \rho_{0}^{1 / 2} B_{0}^{-1} k^{-1}$,

where one must bear in mind that $\xi_{\mathrm{n}}=2-1 / \tilde{\mu}$. These expressions reproduce the behaviour of $\tau_{\mathrm{D}}$ and $\tau_{\mathrm{D}} / P$ with respect to $\tilde{\mu}$ found in Fig. 1.

We now concentrate on the fast wave and study the influence of the magnetic field strength, density and wavenumber on its damping properties, where the frequency is computed from Eq. (24). Given that the fast wave period is independent from the ionisation degree, attention is paid exclusively to $\tau_{\mathrm{D}}$ and $\tau_{\mathrm{D}} / P$. We start with $B_{0}$ (Fig. 2) and see that the fast wave damping time decreases with $B_{0}$, as predicted by Eq. (35). In addition, the most efficient damping, i.e. the smallest $\tau_{\mathrm{D}} / P$, corresponds to the strongest $B_{0}$, also in agreement with Eq. (36).
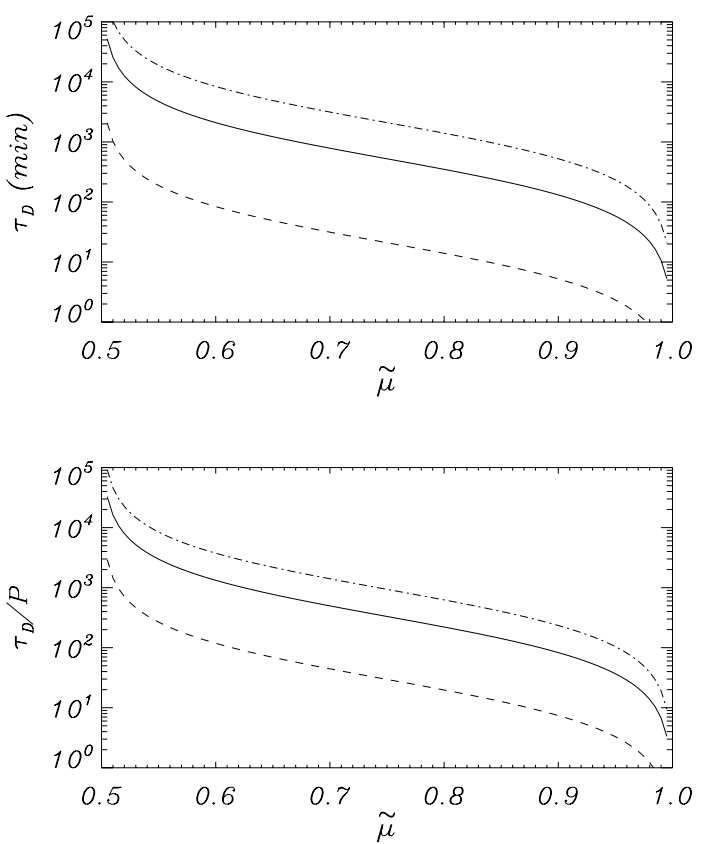

Fig. 3. Fast wave damping time and $\tau_{\mathrm{D}} / P$ versus the ionisation fraction for different values of the density: $\rho_{0}=1 \times 10^{-14} \mathrm{~g} / \mathrm{cm}^{3}$ (dashed), $\rho_{0}=$ $5 \times 10^{-14} \mathrm{~g} / \mathrm{cm}^{3}$ (solid) and $\rho_{0}=1 \times 10^{-13} \mathrm{~g} / \mathrm{cm}^{3}$ (dash-dotted). The other parameters are $T_{0}=8000 \mathrm{~K}$ and $B_{0}=10 \mathrm{G}$, so that the Alfvén and sound speed vary in the ranges $89-282 \mathrm{~km} \mathrm{~s}^{-1}$ and $10.5-14.9 \mathrm{~km} \mathrm{~s}^{-1}$, respectively. Moreover, $k_{x} x_{0}=\pi / 2$ and $k_{z} x_{0}=0.1$.
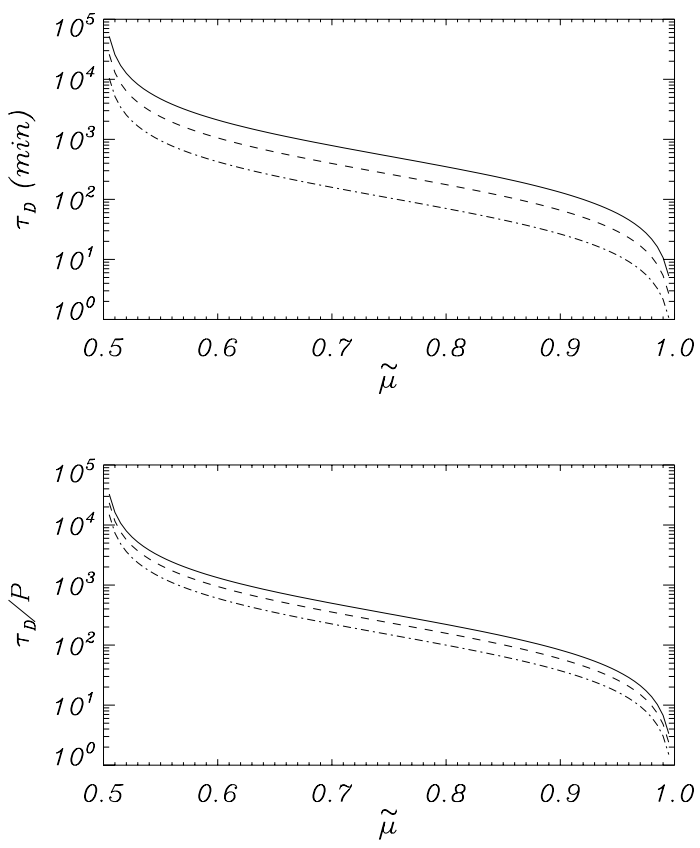

Fig. 4. Fast wave damping time and $\tau_{\mathrm{D}} / P$ versus the ionisation fraction for different values of $k_{z} x_{0}: k_{z} x_{0}=0.1$ (solid), $k_{z} x_{0}=\pi / 2$ (dashed) and $k_{z} x_{0}=\pi$ (dash-dotted). The other parameters are $T_{0}=8000 \mathrm{~K}$, $\rho_{0}=5 \times 10^{-14} \mathrm{~g} / \mathrm{cm}^{3}, B_{0}=10 \mathrm{G}$ and $k_{x} x_{0}=\pi / 2$.

We next study the influence of the density (Fig. 3) and the wavenumber (Fig. 4) in a similar manner. As predicted by Eq. (36), the presence of neutrals in the plasma leads to small $\tau_{\mathrm{D}} / P$ both for small density and/or large wavenumber. Such as happens with the ionisation degree of prominences, these two parameters are not very well known and so there is a large uncertainty about the precise values of $\tau_{\mathrm{D}}$ and $\tau_{\mathrm{D}} / P$ that 

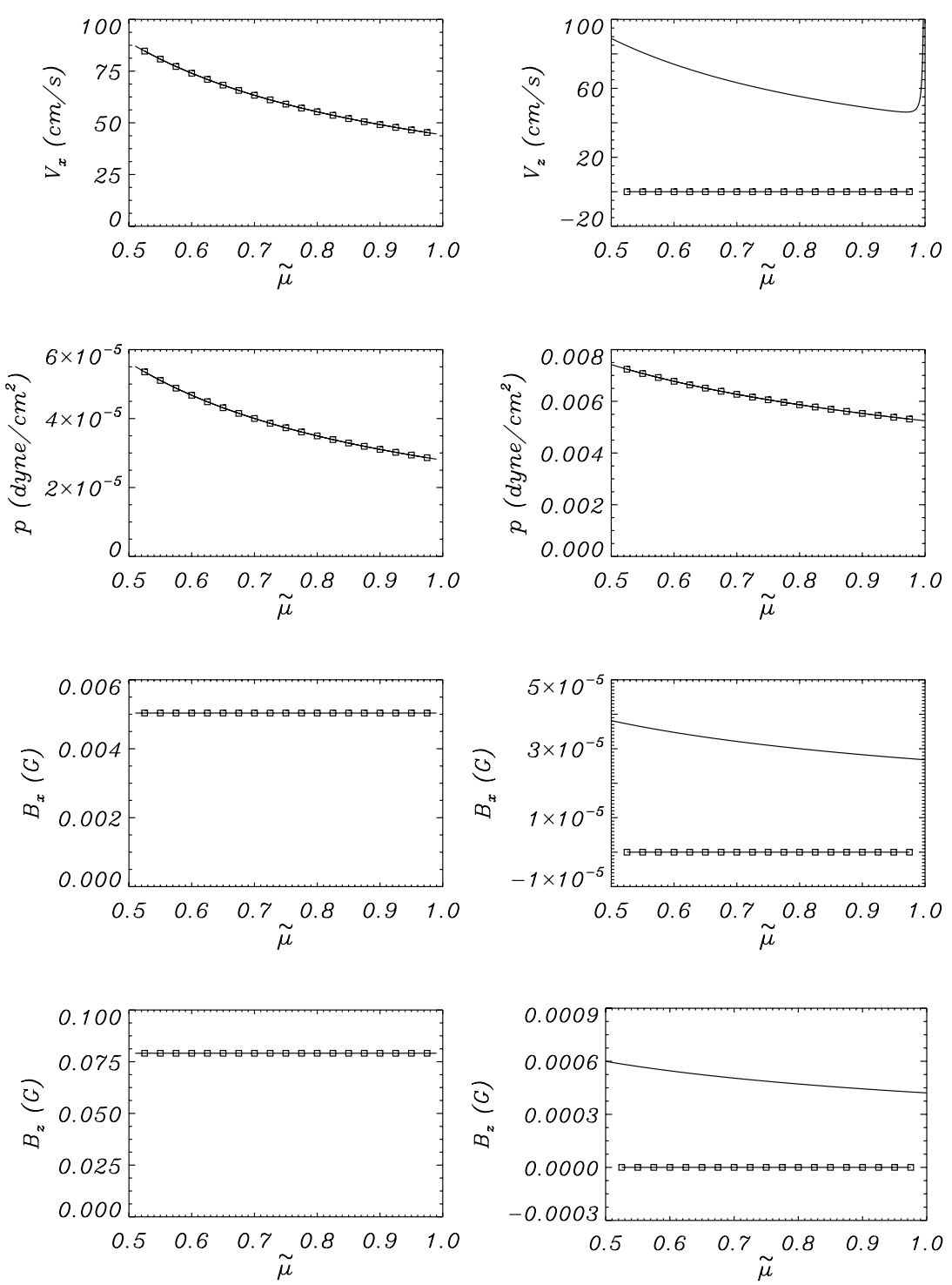

Fig. 5. Perturbations of the velocity, the pressure and the $x$ - and $z$-components of the magnetic field for the fast wave (left) and the slow wave (right). The solid line corresponds to a partially ionised plasma, while squares correspond to a fully ionised, ideal plasma. The perturbed $B_{x}$ and $B_{z}$ for the slow wave in the fully ionised, ideal plasma are several orders of magnitude smaller than the values computed for $\xi_{\mathrm{n}} \neq 0, \eta \neq 0$; for this reason they seem to be zero in the two plots on the right. The parameter values used are $T_{0}=8000 \mathrm{~K}$, $B_{0}=10 \mathrm{G}, \rho_{0}=5 \times 10^{-14} \mathrm{~g} / \mathrm{cm}^{3}, k_{x} x_{0}=\pi / 2$ and $k_{z} x_{0}=0.1$. In addition, for fast waves the arbitrary amplitude $V_{z}=1 \mathrm{~km} \mathrm{~s}^{-1}$ has been taken $\left(V_{x}=1 \mathrm{~km} \mathrm{~s}^{-1}\right.$ for slow waves). can be obtained and, ultimately, about the relevance of the considered mechanism as the cause for the observed damping of prominence oscillations.

Equation (36) predicts that the ratio $\tau_{\mathrm{D}} / P$ of the fast wave is independent of the propagation direction and that only the magnitude of $k$ has some influence on it. To test this prediction we have interchanged the values of $k_{x}$ and $k_{z}$ used in generating Fig. 4. The obtained results are identical to those in Fig. 4, which is the expected behaviour since $k$ remains the same.

\subsection{Perturbations}

The perturbed variables have been computed from Eqs. (31)-(34), with the frequency obtained from Eq. (24), and their moduli have been plotted in Fig. 5. The results include both the solutions for the non-ideal, partially ionised plasma (solid line) and the ideal, fully-ionised plasma (squares). Regarding the fast wave, we find that perturbations coincide for the two cases. Although this may seem a surprising behaviour for values of $\tilde{\mu}$ close to 1 , for which the interactions with neutrals result in the largest modification to the fast wave properties, an explanation can be found from Eq. (33). All the non-ideal terms and the influence of the neutral component of the plasma are concentrated in this formula, but they are absent in the expressions for the other perturbed variables. In addition, the largest contribution in this expression comes from the term with $k_{z} \Xi p$ and, since the pressure perturbation is quite small for fast waves, Eq. (33) results in a $B_{z}$ that is similar for the two cases represented in Fig. 5. Then, since $B_{z}$ does not change much, all other perturbed quantities are similar for the fully ionised ideal case and the partially ionised, non-ideal case. This conclusion remains the same for other propagation angles.

As for the slow wave, the two sets of perturbations are rather different and, again, the pressure perturbation is crucial to understand this behaviour. To plot the slow wave perturbations we have fixed $V_{x}$, so by virtue of Eq. (32) $p$ is fixed to its ideal value (such as found in Fig. 5). Now, the slow wave is characterised by a large pressure perturbation compared to that of the fast wave, so $p$ has an amplification effect on the value of $B_{z}$, and therefore of $B_{x}$, compared to the value represented by squares. This effect is more noticeable for propagation with a large angle with respect to $\boldsymbol{B}_{0}$ because of the presence of $k_{z}$ in the term $k_{z} \Xi p$ in Eq. (33).

Another conclusion one can extract from Fig. 5 is that the inclusion of partial ionisation and non-ideal effects does not influence the predominant polarisation of the magnetoacoustic wave motions since $V_{x} \gg V_{z}$ for slow waves and $V_{z} \gg V_{x}$ for fast waves. 

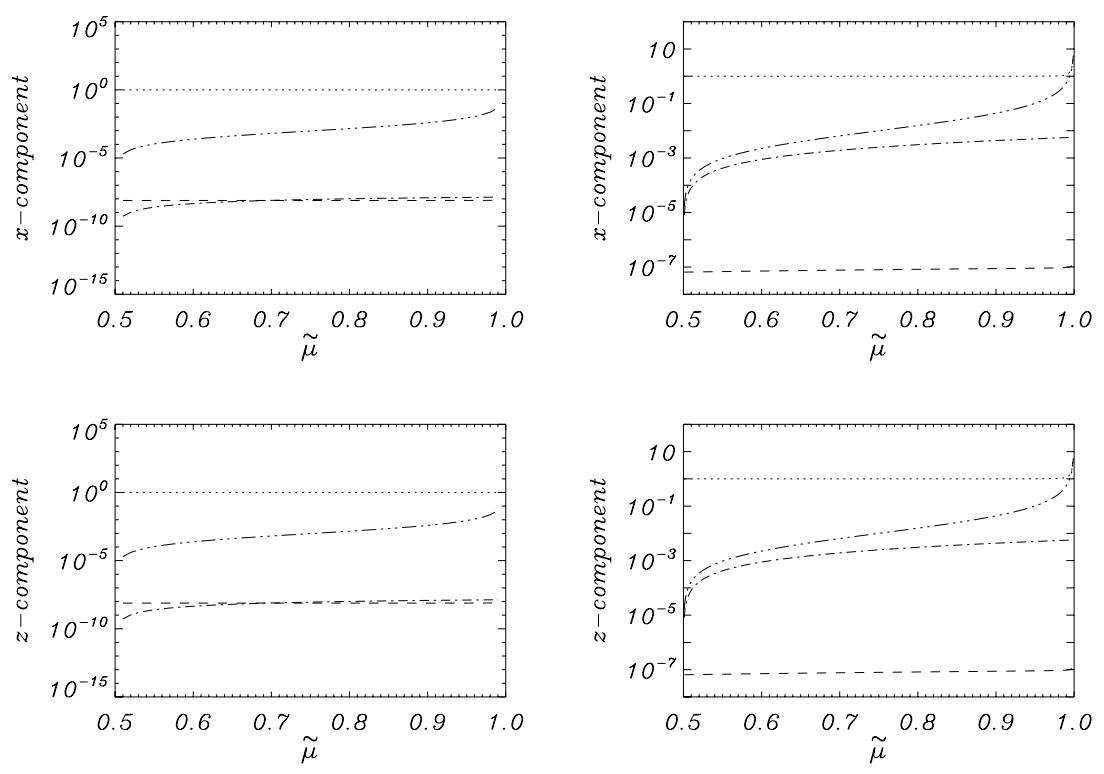

Fig. 6. Importance of the different terms of the induction equation for the fast wave (left) and the slow wave (right). Dotted, dashed, dash-dotted and dashthree-dotted lines correspond to the first, second, third and fourth terms on the right-hand side of Eq. (21). The top and bottom panels correspond to the $x$ - and $z$-components. The parameters used are $T_{0}=8000 \mathrm{~K}$, $\rho_{0}=5 \times 10^{-14} \mathrm{~g} / \mathrm{cm}^{3}, B_{0}=10 \mathrm{G}, k_{x} x_{0}=\pi / 2$ and $k_{z} x_{0}=0.1$.

\subsection{Induction equation}

We now consider the relative importance of the terms in the induction equation and thus plot separately the terms on the right-hand side of the $x$ - and $z$-components of Eq. (21), where the frequency is computed from Eq. (24). These quantities have been normalized by dividing them by the corresponding left-hand side of the equation (i.e. $\mathrm{i} \omega B_{x}$ and $\mathrm{i} \omega B_{z}$, respectively). The results (Fig. 6) show that the second and third terms are negligible in comparison with the other two, that is, in a partially ionised, uniform prominence plasma the induction equation is dominated by the advective and Cowling's diffusion terms. Moreover, advection plays a predominant role for almost the whole range of values of $\tilde{\mu}$ and only when the fraction of neutrals is rather large ( $\tilde{\mu}$ close to 1 ) Cowling's conductivity has an effect comparable to that of advection. These results are almost independent of the propagation direction, although we must emphasise that the present conclusions correspond to prominence conditions.

\section{Comparison with Braginskii (1965)}

Braginskii (1965) gives approximate expressions for the logarithmic damping decrement, $\delta \equiv \omega_{\mathrm{I}} / \omega_{\mathrm{R}}$, of the magnetoacoustic and Alfvén modes in an unbounded medium and in the low $\beta$ limit. The path followed by this author in the derivation of $\delta$ is different from the way that led us to Eq. (24) or Eqs. (29) and (30), so a comparison between both results is necessary. Since we have neglected the viscous and conductive contributions, they are removed from Braginskii's expressions before performing the comparison.

For the fast wave, using Braginskii's Eqs. (8.38), (8.41) and $(8.47 \mathrm{~b})$ we get

$2 \omega_{\mathrm{I}}^{\mathrm{fast}}=\frac{c^{2}}{4 \pi \sigma} k^{2}+\frac{c^{2} k^{2}}{4 \pi} \frac{\xi_{\mathrm{n}}^{2} B_{0}^{2}}{\alpha_{\mathrm{n}} c^{2}}$,

whereas for the slow waves Eqs. (8.38), (8.44) and (8.49) yield

$2 \omega_{\mathrm{I}}^{\text {slow }}=\frac{c^{2}}{4 \pi \sigma} k_{\perp}^{2} \frac{c_{\mathrm{s}}^{2}}{v_{\mathrm{a}}^{2}}+\frac{\rho_{0} c_{\mathrm{s}}^{2} \xi_{\mathrm{n}}^{2}}{\alpha_{\mathrm{n}}}\left[k_{\|}^{2} \frac{n_{\mathrm{i}}^{2}}{n^{2}}+k_{\perp}^{2} \frac{\left(n_{\mathrm{i}}+n_{\mathrm{n}}\right)^{2}}{n^{2}}\right]$.

We rewrite these expressions in our own variables by making the substitutions $k_{\|}=k_{x}$ and $k_{\perp}=k_{z}$ and by also taking into account the definitions in Eq. (2). Then, the former formulae can be written as

$2 \omega_{\mathrm{I}}^{\mathrm{fast}}=\frac{c^{2}}{4 \pi \sigma} k^{2}+\frac{\xi_{\mathrm{n}}^{2} B_{0}^{2}}{4 \pi \alpha_{\mathrm{n}}} k^{2}$

$2 \omega_{\mathrm{I}}^{\text {slow }}=\frac{c^{2}}{4 \pi \sigma} \frac{c_{\mathrm{s}}^{2}}{v_{\mathrm{a}}^{2}} k_{z}^{2}+\frac{\rho_{0} c_{\mathrm{s}}^{2}}{\alpha_{\mathrm{n}}} \frac{\xi_{\mathrm{n}}^{2}}{\left(1+\xi_{\mathrm{i}}\right)^{2}}\left(\xi_{\mathrm{i}}^{2} k_{x}^{2}+k_{z}^{2}\right)$.

Now Braginskii's formulae can be easily compared with our analytical approximations for the imaginary part of the frequency (Eqs. (29) and (30)). It can be appreciated that Eqs. (29) and (39) are identical, although, quite surprisingly, Eqs. (30) and (40) disagree. The difference between these two expressions appears in the second term on the right-hand side, i.e. the one coming from the collisions of electrons and ions with neutrals. To assess the importance of this discrepancy we plot the damping time obtained from the numerical solution of Eq. (24) and from Braginskii's expressions (see Fig. 7). In the case of the fast wave there is a perfect agreement between the two results, as expected. Nevertheless, for the slow wave there is a divergence of $\tau_{D}$ as we move towards parallel propagation $\left(k_{z}=0\right)$. Braginskii's solution is such that for purely parallel propagation the slow wave has a finite damping time, whereas our development leads to no damping of the slow wave for $k_{z}=0$; the cause for this difference is the term proportional to $k_{x}^{2}$ in Eq. (40). Our results make much more sense than Braginskii's since in the limit $k_{z}=0$ the slow wave has $V_{z}=B_{z}=0$ and thus the terms responsible for the damping vanish (see Eqs. (31)-(34)). Hence, it is clear that the slow wave cannot be damped in this limit, such as displayed by our results in Fig. 7 .

We finally note the smaller but noticeable difference in $\tau_{\mathrm{D}}$ for the slow wave and $k_{z} \geq 1$ in Fig. 7, whose origin lies in the factor $1 /\left(1+\xi_{\mathrm{i}}\right)$ on the right-hand side of Eq. (30) and which incorrectly appears as $1 /\left(1+\xi_{\mathrm{i}}\right)^{2}$ in Eq. (40).

\section{Conclusions}

In this work we give a succint derivation of the one-fluid MHD equations for a partially ionised plasma. We next study the temporal damping of the magnetoacoustic waves in a partially ionised prominence plasma considering an equilibrium model 

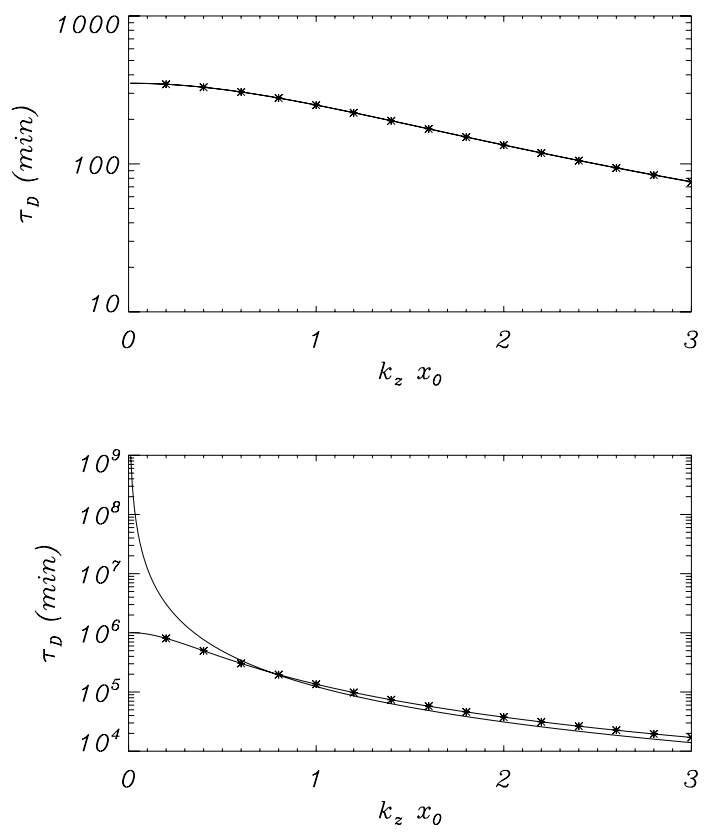

Fig. 7. Damping time obtained with our dispersion relation (Eq. (24)) (solid line) and with Braginskii's formulae (symbols) for the fast and slow waves (top and bottom panels, respectively). These results have been obtained with the parameter values $T_{0}=8000 \mathrm{~K}, B_{0}=10 \mathrm{G}$, $\rho_{0}=5 \times 10^{-14} \mathrm{~g} / \mathrm{cm}^{3}, \tilde{\mu}=0.8$ and $k_{x} x_{0}=\pi / 2$.

with uniform magnetic field (i.e. without electric current). Linear perturbations have been assumed. The conclusions of this work (which are only strictly applicable to prominence conditions) can be summarised as follows:

- We have found that the damping of MHD waves by ionneutral interactions is much stronger for the fast wave than for the slow wave, unlike what happens with other damping mechanisms, such as radiative cooling and conduction, for which the slow wave is strongly attenuated while the fast wave remains almost undamped (Terradas et al. 2002; Carbonell et al. 2004; Terradas et al. 2005). A consequence of the present and previous studies is that the observed damped oscillations in prominences can be explained by slow waves attenuated by radiative cooling and conduction or by fast waves attenuated by ion-neutral collisions.

- We have studied the dependence of the fast wave damping time with the different equilibrium parameters.

a) As expected, the importance of collisions between ions and neutrals grows with the proportion of neutrals in the plasma. This means that it is more relevant for small ionisation fractions and that it can be neglected for nearly fully ionised, or completely ionised, plasmas.

b) Equation (36) constitutes a good approximation for the fast wave ratio of damping time to period, $\tau_{\mathrm{D}} / P$. This formula indicates that the strongest damping (i.e. the smallest $\tau_{\mathrm{D}} / P$ ) occurs for strong magnetic fields, small densities and small ionisation fractions. Given the poor knowledge about the values of the last two parameters in prominences, it is hard to judge whether the physics of partial ionisation are relevant in these solar objects and whether it can be the cause for the damping of fast waves in solar prominences, although it is not possible either to discard its importance. It is worth stressing that although this mechanism has a noticeable effect on the damping of fast MHD waves, it only yields acceptable damping times for relatively large values of the ionisation fraction.

- The fast wave perturbations are not influenced by the inclusion of non-ideal mechanisms and partial ionisation. On the other hand, the slow wave is characterised by having larger magnetic field perturbations than in the ideal, fully ionised case. Moreover, the characteristic velocity polarisation of the two magnetoacoustic waves (along and across the magnetic field for slow and fast waves, respectively) is maintained when the effects of neutrals and collisions are taken into account.

- Regarding the importance of the various terms in the induction equation, our results show that the advective term is dominant over the whole range of values of $\tilde{\mu}$ and that for $\tilde{\mu} \sim 1$ (i.e. small ionisation) Cowling's diffusion also must be taken into account.

- From the magnetoacoustic wave dispersion relation we have obtained an analytical approximation for the real and imaginary parts of the frequency that perfectly matches the exact results. This analytical approximation has been compared with the formulae provided by Braginskii (1965) and a discrepancy between them has been found. The most important difference between the two sets of formulae is the term proportional to $k_{x}^{2}$ in Braginskii's formula for the slow wave damping time, which (according to Braginskii) results in the damping of this wave for propagation nearly parallel to the unperturbed magnetic field. Since the slow wave cannot be damped when $k_{z}=0$, we conclude that Braginskii's expression for $\omega_{\mathrm{I}}^{\text {slow }}$ contains a physical inconsistency in this limit. The origin of this disagreement must lie in the different procedure used to describe the perturbations: while we have chosen to perturb all physical variables and to insert them into the basic equations for the partially ionised plasma (Eqs. (4)-(6) and (14)), Braginskii makes use of the plasma entropy (see Eqs. (6.38), (6.39) and (8.37) in Braginskii 1965). We then infer that Braginskii's treatment fails to describe the slow wave damping by collisions with neutrals for nearly parallel propagation.

It has been noted that the equilibrium used in this work has no electric currents, which, together with the linear assumption, has the consequence of removing the effect of Joule dissipation from the energy equation. In addition, in the absence of electric currents the support mechanism of the prominence material against gravity cannot be properly incorporated. It can thus be interesting to study the influence of equilibrium electric currents and gravity on the damping of the magnetoacoustic waves. The limitations imposed by the finite size of prominences and by the surrounding coronal plasma will also be included in a future work.

Acknowledgements. The authors thank T. Arber and J. Leake for fruitful discussions during their stay in the Space Research Institute of Graz, whose staff is also acknowledged for their kind hospitality. P. Forteza thanks the Spanish Ministry of Education and Science for a fellowship. M. Khodachenko acknowledges the Austrian Fonds zur Förderung der wissenschaftlichen Forschung (Project P16919-N08). The authors have benefited of financial support from the Austrian Governement and the Spanish Ministerio de Educación y Ciencia through the ÖAD-Acciones Integradas programme (project No. 11/2005 and HU2004-0010). The authors also acknowledge the financial support received from MCyT AYA2003-00123. 


\section{References}

Anzer, U., \& Heinzel, P. 2005, ApJ, 622, 714

Ballai, I. 2003, A\&A, 410, L17

Braginskii, S. I. 1965, Rev. Plasma Phys., 1, 205

Carbonell, M., Oliver, R., \& Ballester, J. L. 2004, A\&A, 415, 739

Chiuderi Drago, F. 2005, A\&A, 443, 1055

Engvold, O. 2001, in INTAS Workshop on MHD Waves in Astrophysical Plasmas, ed. J. L. Ballester, \& B. Roberts (Spain: Universitat de les Illes Balears), 123

Gilbert, H. R., Hansteen, V. H., \& Holzer, T. E. 2002, ApJ, 577, 464

Goedbloed, J. P. H., \& Poedts, S. 2004, Principles of Magnetohydrodynamics (CUP)

Heinzel, P., Anzer, U., \& Gunár, S. 2005, A\&A, 442, 331
Khodachenko, M. L., Arber, T. D., Rucker, H. O., \& Hanslmeier, A. 2004, A\&A, 422, 1073

Khodachenko, M. L., Rucker, H. O., Oliver, R., Arber, T. D., \& Hanslmeier, A. 2006, Adv. Space Res., 37, 447

Labrosse, N., \& Gouttebroze, P. 2004, ApJ, 617, 614

Leake, J. E., \& Arber, T. D. 2006, A\&A, 450, 805

Leake, J. E., Arber, T. D., \& Khodachenko, M. L. 2005, A\&A, 442, 1091

Oliver, R. 1999, in ESA SP-448: Magnetic Fields and Solar Processes, ed. A. Wilson, et al., 425

Oliver, R., \& Ballester, J. L. 2002, Sol. Phys., 206, 45

Patsourakos, S., \& Vial, J.-C. 2002, Sol. Phys., 208, 253

Terradas, J., Carbonell, M., Oliver, R., \& Ballester, J. L. 2005, A\&A, 434, 741

Terradas, J., Molowny-Horas, R., Wiehr, E., et al. 2002, A\&A, 393, 637

Terradas, J., Oliver, R., \& Ballester, J. L. 2001, A\&A, 378, 635 